\title{
"KNOB" AT THE PERIPHERY OF DESCEMET'S MEMBRANE *
}

BY

\section{ARNOLD LOEWENSTEIN}

T'nnent Institute for Ophthalmology, University of Glasgow. Director, Prof. Riddell.

As is well-known the peripheral end of Descemet's membrane in antero-posterior sections usually tapers to a point. This is shown clearly at $a$ in Fig. 1. What has not been previously described

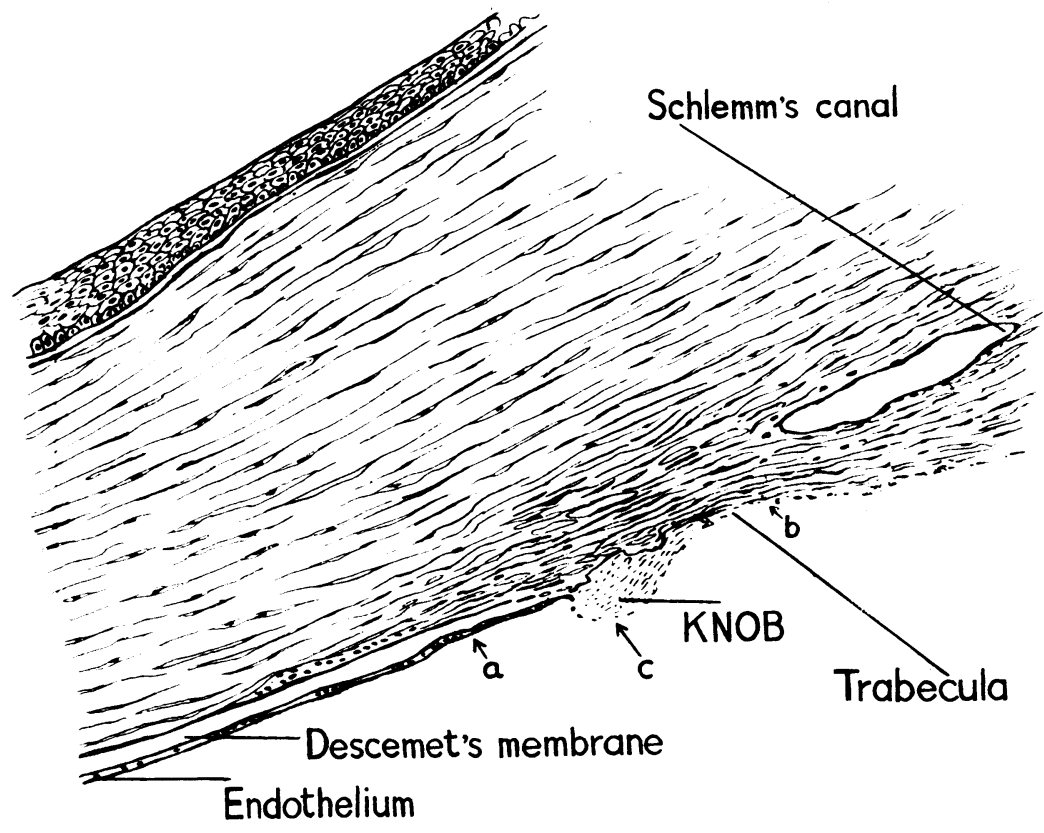

FIG. 1.

however is that the usually flat tissue between a and the trabecula (b) may project backwards as a knob (c). This swelling is granular and does not contain cells (Figs 2 and 3 ).

Here this knob-like bulge has been demonstrated in five eyes, three knobs were normal and two were malignant melanomata. Generally, the five cases (Figs 2-6), show the different structure of the knob, the prominent zone in the area of the gap.

Ve understand that the aqueous fluid enters the gap and reaches the posterior lamellae. The aqueous does not filter the posterior

\footnotetext{
* Received for publication November 10, 1949.
} 


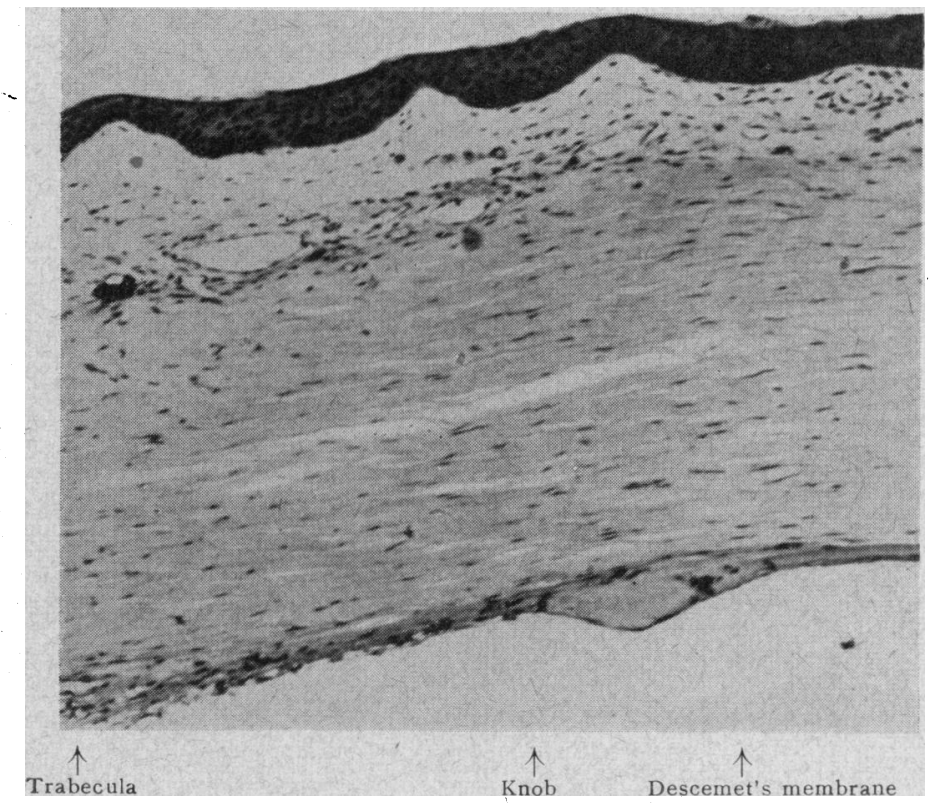

FIG. 2.-Prominent Schwalbe's ring. The Knob is bulged without lamellae. Some dark nuclei in the endothelium. (H.E. 300 ×.)

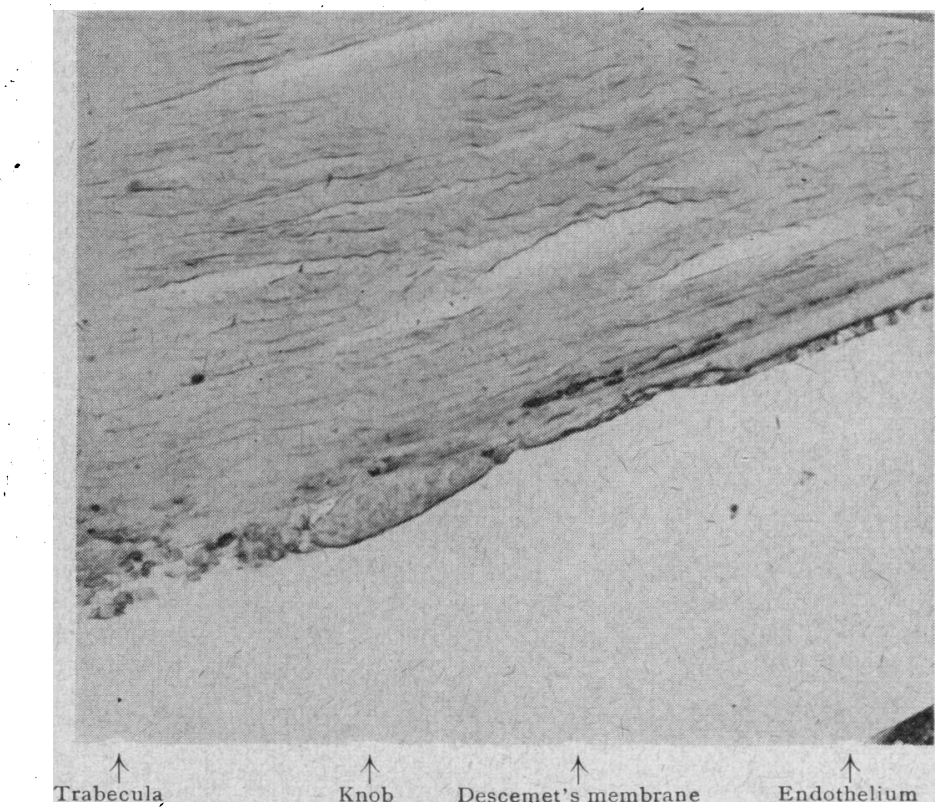

FIG. 3.-Malignant choroidal melanoma. Prominent Schwalbe's ring. Note the granular structure of its tissue. (Azanst. $300 \times$.) 
lamellae as the end of Descemet is completely intact (Fig. 1,a). On the other hand, the aqueous passes the meshwork of the trabeculae and finally reaches Schlemm's canal (Fig. 1,b). The important gap is between $a$ and $b$. In five sections the tissue in the gap shows the swollen knob as at $c$ in Fig. 1 in the prominent area.

The aqueous enters the gap and may produce different reactions :

(1) In some eves the posterior lamellae in the gap may remain

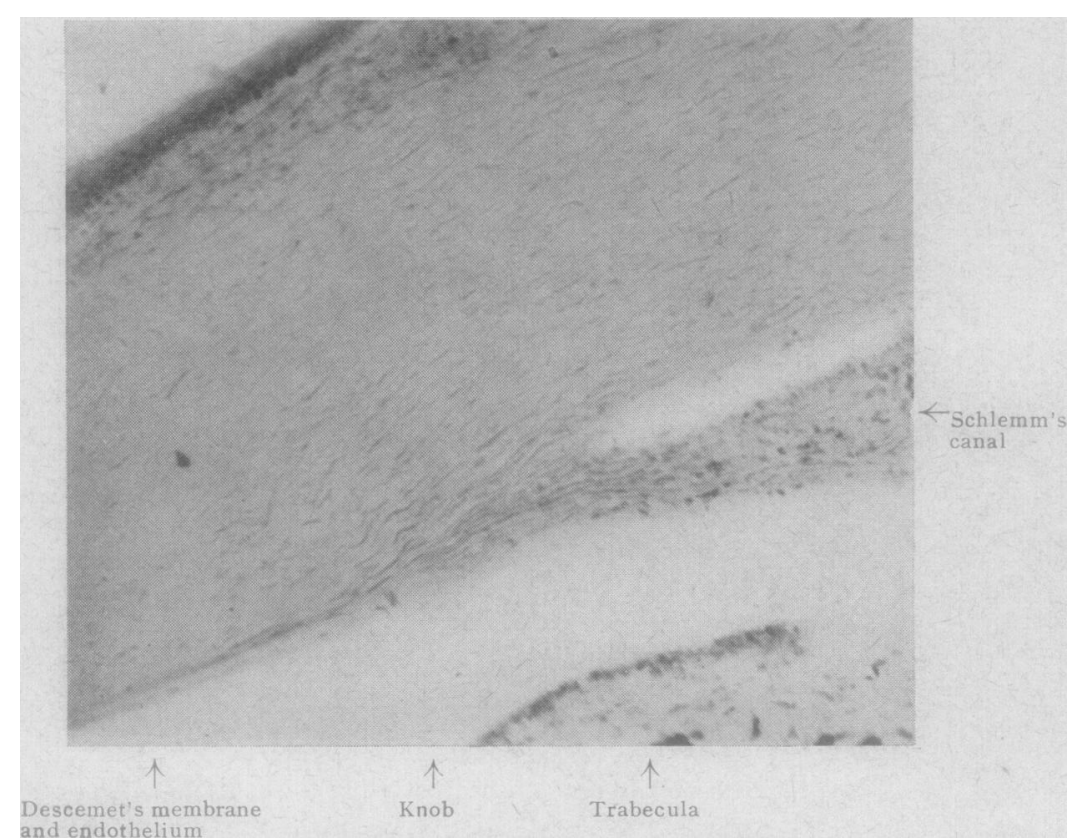

FIG. 4.-Typical Knob between end of Descemet's membrane and Trabecula. (H.E. $300 \times$.)

flat and form the white ring of the excised eye seen with the slitlamp. This is identical with the white ring of Schwalbe, and is well known in clinical gonioscopy.

(2) In other eyes the posterior lamellae may be swollen to form the prominent white ring seen with a high-power slit-lamp.

It is not easy to prove the histological appearance of Schwalbe's ring in section. It seems that the aqueous fluid does not produce characteristic changes detectable on staining. On the other hand, the prominent knob is perfectly seen in Figs 2-4.

We assume the aqueous enters the gap between $a$ and $b$ and swells the posterior lamellae to form the whitish ring. The whitish ring was demonstrated at the Oxford Congress (Loewenstein, 1948), en face and in profile in Donald's original picture. The 
whitish ring in the slit-lamp and the prominent knob may be identical. A multiple congenital anomaly of the eye discussed by A. J. Ballantyne (1949) from another point of view, shows a prominent nodule in the area of the gap between the end of the Descemet and the trabecula (Fig. 5).

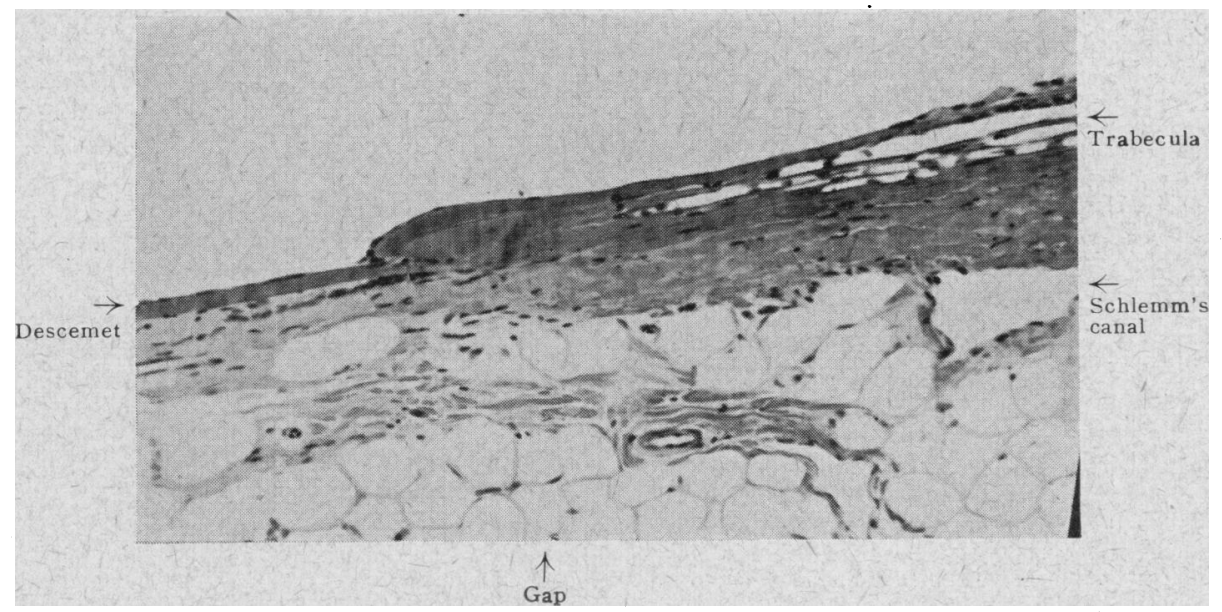

FIG. 5.-Congenital anomaly. Nodule between end of Descemet's membrane and trabecula. (Van Giesson. $300 \times$.)

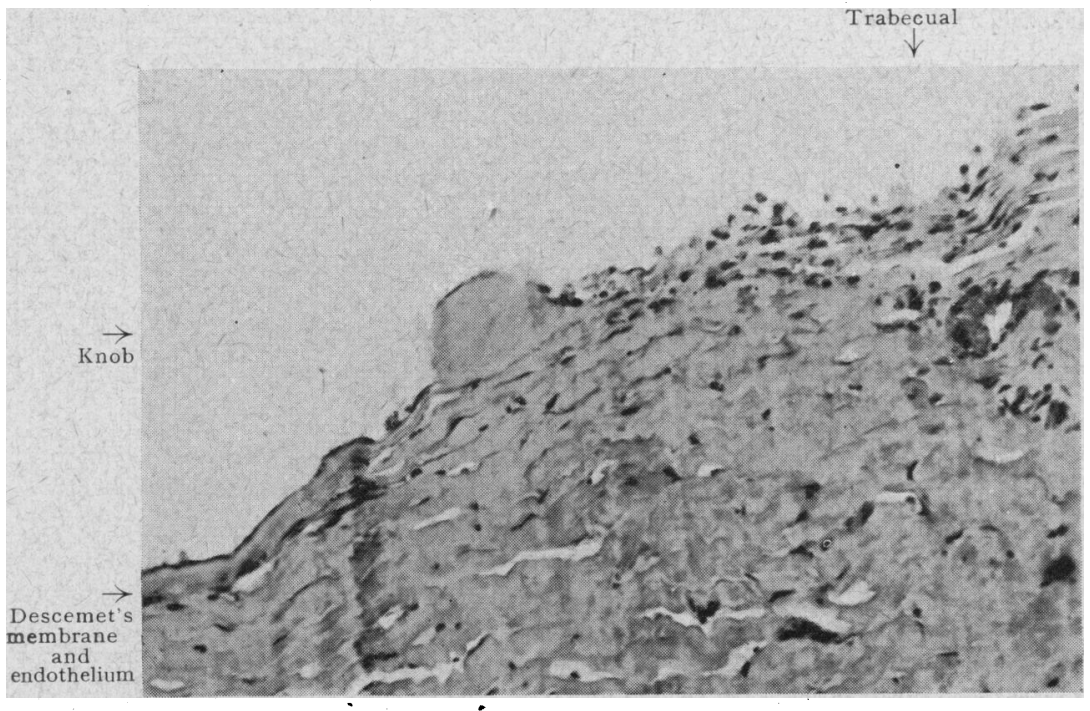

Fig. 6.-Knob-like bulge in area between Descemet's end and trabecula. Mustard gas traumatism. (H.E. $300 \times$.) 
Both processes seem to be physiological. But some pathological changes may occur. I have observed a case of corneal degeneration in an eye injured by mustard gas in the first world war about 30 years ago. (The case is to be published by Dr. Thakore.) The corneal lamellae were intensely swollen and dilated, with many huge vessels surrounded with lymphatic infiltration. A knob bigger than in the other cases was prominent (Fig. 6). The gap of the area between $a$ and $b$ was exactly the same. This knob contained cloudy tissue without any nuclei. I am not sure whether the nodule in Fig 6 had the same physiological characteristics, in which case it might be that the lamellae had been damaged by some chemical or other influence.

\section{Summary}

The changes in the whitish ring of Schwalbe seen in the excised eye with the slit-lamp have been explained anatomically. In five cases the typical prominent knob has been demonstrated. Schwalbe's ring and the knob appear to be the same.

\section{REFERENCES}

Ballantyne, A. J. (1949). Proc, roy. Soc. Med., 42, 756.

Loewenstein, A. (1948). Trans, ophthal. Soc. U.K., 68, 488, fig. 157.

\section{NOTES}

Toronto

THE Department of Ophthalmology in the University of Toronto has received a grant from the Ontario Provincial Department of Health through funds provided by federal grant for research work on the prevention of blindness from glaucoma. This work will be done under the direction of Dr. T. H. Hodgson in the Banting Institute, Toronto General Hospital, and other University teaching hospitals in Toronto. The initial grant was $\$ 8,200$. It is proposed that the research work will extend over 3 to 5 years. 